Classification

\title{
HREM Observations of Ion Bombardment-Induced Dislocation Loops in $\mathrm{ZnO}$
}

\author{
Jean-Jacques Couderc $\left({ }^{1}\right)$, Jean-Jacques Demai $\left({ }^{2}\right)$, Guy Vanderschaeve $\left({ }^{1}\right)$ and Alain \\ Peigney $\left({ }^{2}\right)$ \\ ( $\left.{ }^{1}\right)$ Laboratoire de Physique des Solides, ERS CNRS 111, INSA, Complexe Scientifique de Rangueil, \\ 31077 Toulouse Cedex, France \\ ${ }^{2}$ ) Laboratoire de Chimie des Matériaux Inorganiques, ERA CNRS 1311, Université Paul Sabatier, \\ 118 Route de Narbonne, 31062 Toulouse Cedex, France
}

(Received February 24; accepted April 10, 1995)

Résumé. - On utilise la microscopie électronique en haute résolution pour mettre en évidence des boucles de dislocations partielles provenant de l'amincissement par bombardement ionique d'un échantillon de $\mathrm{ZnO}$. Les défauts d'empilement créés par insertion d'une double couche de $\mathrm{Zn}$-O dans le plan basal sont du type $1 \Delta$.

\begin{abstract}
High resolution electron microscopy (HREM) is used to show faulted dislocation loops resulting from ion bombardment thinning in $\mathrm{ZnO}$. The stacking faults are created by the insertion of a $\mathrm{Zn}-\mathrm{O}$ double layer in the basal plane and are of the $1 \Delta$ type (complex stacking fault).
\end{abstract}

\section{Introduction}

High resolution electron microscopy (HREM) investigations of lattice defects in II-VI compounds with the wurtzite structure have already been performed on $\mathrm{CdS}$ [1-4], $\mathrm{ZnO}$ [5-7] and $\mathrm{CdSe}$ strained at room temperature [4]. In the latter material thinned by argon ion milling (beam intensity $250 \mu \mathrm{A}$, accelerating voltage $5 \mathrm{kV}$ ), interstitial Frank loops were frequently observed; they were considered as having been introduced during the ion thinning process as a result of damage by the ion bombardment.

In the course of a systematic investigation of the microstructure of $\mathrm{ZnO}$-based compounds that are base-constituent of ceramic semiconductor devices (varistors), we have observed that ion bombardment may introduce lattice defects in the thin foil samples. The present paper reports on the results of a HREM study of the irradiation-induced faulted dislocation loops in a doped $\mathrm{ZnO}$ crystal with wurtzite structure. The samples have a global cationic composition $\mathrm{Zn}_{97.4} \mathrm{Bi}_{1} \mathbf{M n}_{1} \mathrm{Ti}_{0.6}$. They were made by sintering at $950^{\circ} \mathrm{C}$ followed by air quenching. $\mathrm{Bi}$ and $\mathrm{Ti}$ atoms are standard 
added elements favouring liquid phase sintering and grain growth, respectively. Mn atoms are added to improve the electrical properties of the compound.

Thin foils suitable for transmission electron microscopy (TEM) were made in the following way: $300-400 \mu \mathrm{m}$ thick slices were cut with a wire saw and then mechanically ground down to $50 \mu \mathrm{m}$; the final thinning was performed by argon ion milling (beam intensity $10 \mu \mathrm{A}$, accelerating voltage $6 \mathrm{kV}$ ). The preparation of thin samples by chemical methods was unsuccessfull.

The observations were carried out on a JEOL 2010 electron microscope operated at $200 \mathrm{kV}$ and fitted with a nanoanalysis EDX equipment (TEM-SCAN service of the Universite Paul Sabatier, Toulouse).

\section{Stacking Faults in the Wurtzite Structure}

A brief description of the wurtzite structure and the different types of stacking faults in this structure is now given.

The wurtzite structure can be represented as a stacking of double layers: aAbBaAbBaAbB (or $\Delta \nabla \Delta \nabla \Delta \nabla \Delta \nabla$ in the Frank notation), packed normal to the [0001] direction. Each layer is composed of one atom species.

Three types of stacking faults are possible in the basal plane:

i) The intrinsic fault is produced as a result of a slip of a partial Shockley dislocation $1 / 3\langle 01 \overline{1} 0\rangle$ in the basal plane so that the double layers aA transform into $\mathrm{cC}$, and $\mathrm{bB}$ into aA. The faulted sequence is: $\mathrm{aAbBaAbBcCaAcCaAcC}$ or $\Delta \nabla \Delta \Delta \Delta \nabla \Delta \nabla \Delta(2 \Delta$ fault or intrinsic stacking fault according to [4]).

ii) The extrinsic fault is generated by the insertion of a $\mathrm{cC}$ double layer so that the stacking sequence is: $\mathrm{aAbBaAbBcCaAbBaAbB}$ or $\Delta \nabla \Delta \nabla \Delta \Delta \Delta \Delta \nabla \Delta \nabla$ (3 $\Delta$ fault or extrinsic stacking fault). It is bounded by a pure Frank partial dislocation $b=1 / 2[0001]$.

iii) The $1 \Delta$ fault (or complex fault according to [4]) corresponds to the following stacking sequence: aAbBaAbBcCbBcCbBcC or $\Delta \nabla \Delta \Delta \nabla \Delta \nabla \Delta \nabla$. As shown in [8], it can be produced either by growth, or by a two-stage mechanism (interstitial defect):

1) A two-atom layer is inserted into the $\mathrm{cC}$-position, creating an extrinsic fault bounded by a loop whose Burgers vector is $1 / 2[0001]$.

2) The loop is swept by a single Shockley partial $(b=1 / 3\langle 01 \overline{1} 0\rangle)$ leaving a complex fault with displacement vector $\mathbf{R}=1 / 2[0001]+1 / 3\langle 01 \overline{1} 0\rangle=1 / 6\langle 02 \overline{2} 3\rangle$. The $3 \Delta$ stacking fault is transformed into a single $1 \Delta$ complex fault. A similar mechanism is involved for the generation of $1 \Delta$ vacancy loops.

Notice that both $1 \Delta$ and $3 \Delta$ faults are bounded by dislocation loops whose Burgers vectors $(1 / 6\langle 02 \overline{2} 3\rangle$ and $1 / 2[0001]$ respectively) do not lie in the fault plane. The Burgers vector lengths are in the ratio $\left(c^{2} / 4+a^{2} / 3\right)^{1 / 2} /(c / 2)$, i.e. 1.23 in the case of $\mathrm{ZnO}(a=0.3249 \mathrm{~nm}, c=$ $0.52047 \mathrm{~nm})$.

The relative energies of the various types of stacking faults are expected to be roughly proportional to the number of wrong second neighbour stackings; therefore the approximative stacking fault energy ratio is $1 / 2 / 3$ for the $1 \Delta, 2 \Delta$ and $3 \Delta$ stacking faults, respectively. Consistent with this view, in CdSe [4], interstitial type Frank loops were frequently observed to nucleate on wide intrinsic stacking faults generated by plastic deformation, thus transforming a $2 \Delta$ fault into a $1 \Delta$ one. 


\section{Observations}

Conventional TEM observations revealed the presence of both intergranular micrometric phases $\left(\beta-\mathrm{Bi}_{2} \mathrm{O}_{3}\right.$ and $\left.\mathrm{Zn}_{2} \mathrm{TiO}_{4}\right)$ and intragranular nanometric precipitates (including $\mathrm{Mn}$ and $\mathrm{Ti}$ atoms) [9]. In the course of a HREM investigation which was primarily intended to study the structure and coherency of the latter ones, faulted loops, whose diameter ranges from 3 to $12 \mathrm{~nm}$ were observed. They are viewed edge-on in Figure 1 and appear as fine dark or light lines. The density of loops is very high in the thinnest part of the foil (about $10^{14-15} / \mathrm{mm}^{3}$ ). So small features were hardly distinguished using conventional dark field techniques, owing to the surface damage due to ion beam thinning. On the other hand EDX analysis (probe size $1.5 \mathrm{~nm}$ ) inside the loops did not reveal the presence of any impurities. So it is likely that the loops do not result from any segregation of $\mathrm{Bi}, \mathrm{Mn}$ and $\mathrm{Ti}$ doping atoms.

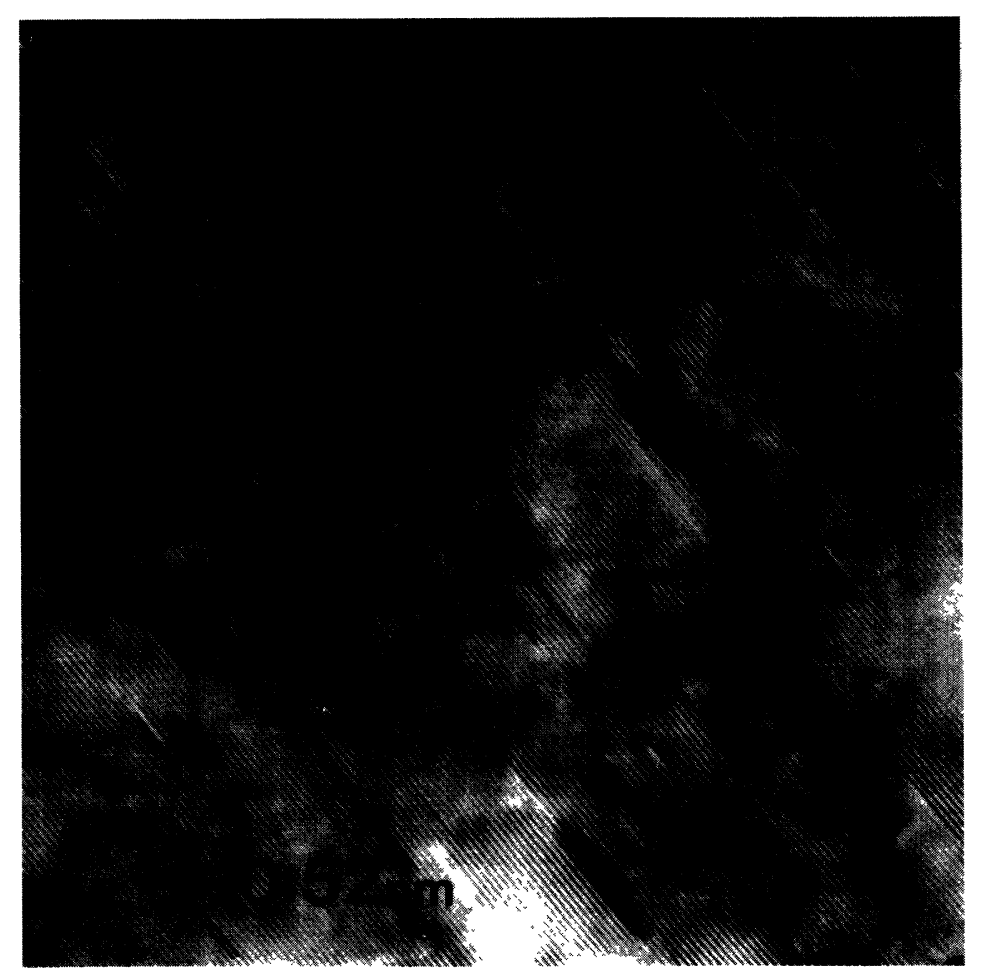

Fig. 1. - Lattice image taken with the $\pm 0001, \pm 0002$ diffracted waves of an area with a high density of faulted loops. The electron beam is parallel to $[2 \overline{1} \overline{1} 0]$. The spacing of the lattice fringes $(0.52 \mathrm{~nm})$ corresponds to the interplanar distances between basal planes $\left(d_{0001}\right)$.

Figure 2 shows an area free from defects: the HREM image consists of rows of white spots that are slightly in zig-zag in the $c$ direction. As shown in [3] each bright spot corresponds to a close pair of zinc and oxygen atomic rows.

Figure 3 shows an insertion dislocation loop. One can see that the aAbB stacking of the wurtzite structure is modified into aAbBaAbBcCbBcCbBcC $(\Delta \nabla \Delta \nabla \Delta \Delta \nabla \Delta)$, that is a $1 \Delta$ stacking fault, 

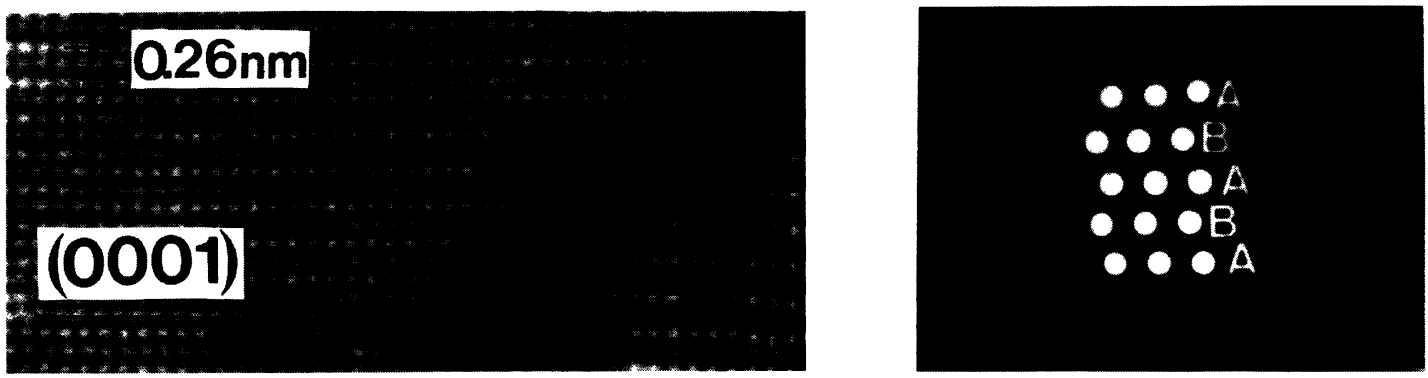

Fig. 2. - a) HREM micrograph of an area free from defects taken with the electron beam parallel to [21 $\overline{1} 0]$ using 16 diffracted waves. b) Schematic representation of the stacking sequence in the [0001] direction.

the partial dislocations that limit the fault on both side having $b=1 / 6\langle 02 \overline{2} 3\rangle$. All the faulted loops investigated were found to be of the same type.

\section{Discussion}

As mentioned above, the presence of these defects cannot be attributed to the segregation of $\mathrm{Mn}$, $\mathrm{Bi}$, Ti impurities. It is suggested that the insertion loops have probably been produced during the ion bombardment that would promote a migration and a condensation of interstitial atoms (both $\mathrm{Zn}$ and $\mathrm{O})$.

The following discussion will focus on two main points of the present study: the occurrence of $1 \Delta$ loops and a comparison in the defect structure resulting from either ion bombardment or electron irradiation.

4.1 WHY ARE ONLY $1 \Delta$ LOOPS OBSERVED?. - In our experiments only $1 \Delta$ faulted loops were observed. As mentioned above, these defects are generated firstly by inserting a double layer of $\mathrm{Zn}$ and $O$ interstitial atoms and subsequently transforming the so-created $3 \Delta$ fault into a lower energy $1 \Delta$ one by nucleation of a Shockley partial loop. At first sight, the present observations could be accounted for by comparing the relative energies of $1 \Delta$ and $3 \Delta$ stacking faults. Nevertheless, assuming the defects ( $1 \Delta$ and $3 \Delta$ faulted loops) are in their equilibrium configuration, a discussion of their relative stability should compare their total energy, which includes both the surface energy of the stacking faults and the line energy of the dislocation loops. Calculations (see appendix) indicate that if $\gamma_{3 \Delta}-\gamma_{1 \Delta}$, which is roughly equal to $\gamma_{2 \Delta}$ ( $\gamma$ : Stacking fault energy), is higher than a critical value $\gamma_{c}, 1 \Delta$ loops are stable whatever their size; if $\gamma_{3 \Delta}-\gamma_{1 \Delta}$ is lower than $\gamma_{c}$ only loops with radius $r$ lower than a critical value $r_{\mathrm{c}}$ are stable in their $3 \Delta$ form. Note that both $\gamma_{\mathrm{c}}$ and $r_{\mathrm{c}}$ strongly depend on the core radius chosen in the calculation; $\gamma_{\mathrm{c}}$ is certainly higher than $370 \mathrm{~mJ} / \mathrm{m}^{2}$. Unfortunately, the fault energies in $\mathrm{ZnO}$ are not precisely known so that no definite conclusions can be drawn. Consistent with the previous results of Takeuchi et al. [10], we were not able to observe dissociated perfect dislocations in this compound, even when using dark field weak beam imaging. Accordingly, only a lower limit of $\gamma_{2 \Delta}$ can be estimated: $\gamma_{2 \Delta}>43 \mathrm{~mJ} / \mathrm{m}^{2}$ [10].

It was previously shown that ion bombardment induced the formation of $1 \Delta$ and $3 \Delta$ faults in CdSe with wurtzite structure [4]. As mentioned above, $1 \Delta$ faults were observed to form by nucleation of Frank loops on preexisting glide stacking faults. Apparently, in the specimen areas free from wide intrinsic stacking faults, only $3 \Delta$ faulted loops were observed, at variance with 

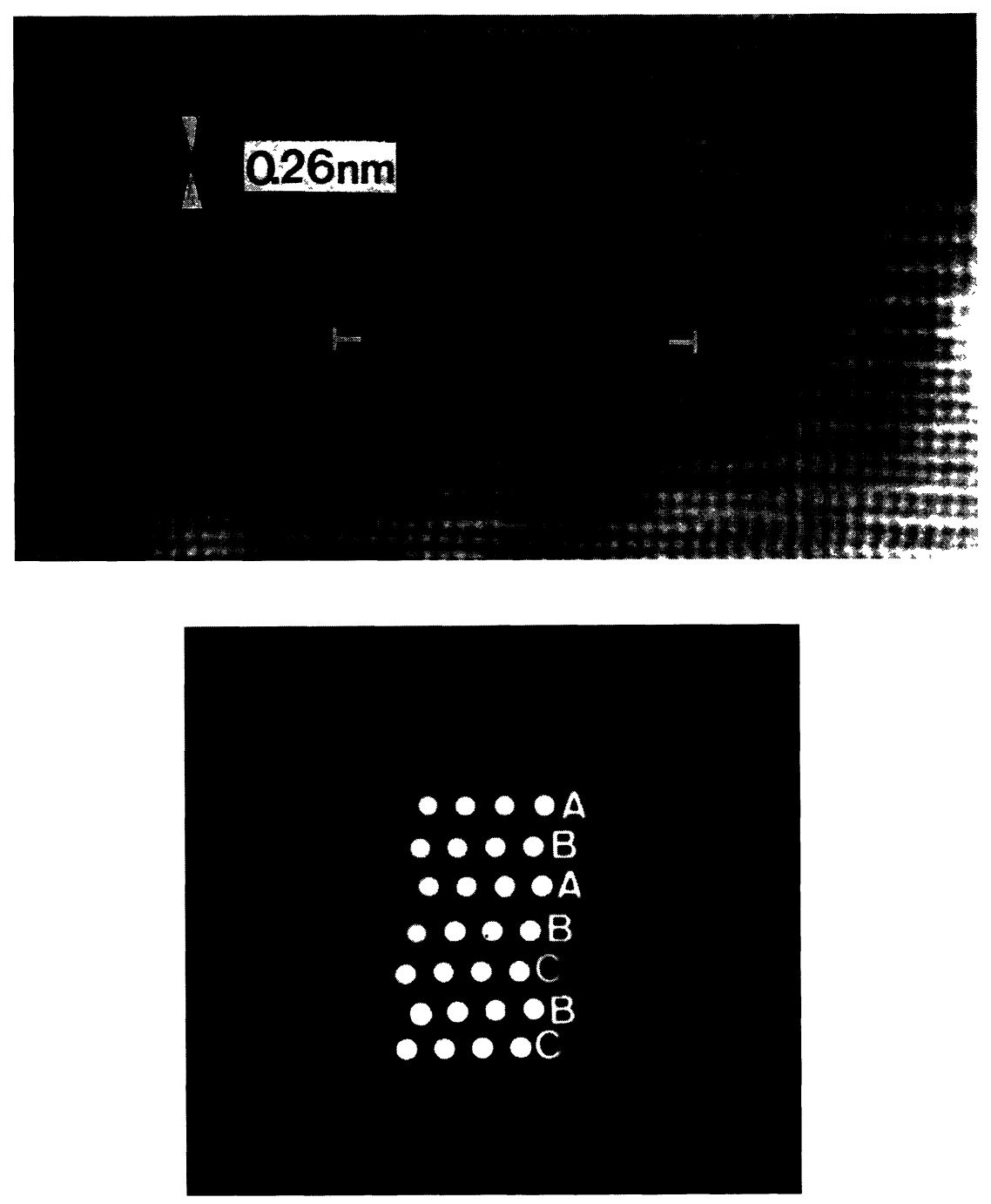

Fig. 3. - a) HREM image of a complex $1 \Delta$ fault (same conditions as Fig. 2a). b) Schematic representation of the stacking sequence of a $1 \Delta$ stacking fault.

the present results in $\mathrm{ZnO}$. This could be related to the rather low value of $\gamma_{2 \Delta}$ in CdSe: $\gamma_{2 \Delta}=$ $14 \pm 5 \mathrm{~mJ} / \mathrm{m}^{2}[4,10]$.

4.2 COMPARISON WITH ELECTRON IRRADIATION EXPERIMENTS. - Another way to produce interstitial extended defects in $\mathrm{ZnO}$ is electron irradiation with a high voltage electron microscope. It has been shown elsewhere that dislocation loops nucleate both on preexisting stacking fault planes and in regions free from stacking faults as a result of irradiation with $700 \mathrm{keV}$ electrons. In the latter regions two types of interstitial loops were identified: perfect loops lying in the prismatic planes and $3 \Delta$ faulted loops lying in the basal plane. No $1 \Delta$ faulted loops were observed in these regions [11-14]. 
The difference in the defect structure of $\mathrm{ZnO}$ resulting from either ion bombardment (present work) or high energy electron irradiation [11-14] is not fully understood. According to Yoshiie et al. [13-14], the loop nucleation process in semiconducting crystals is an heterogeneous process with some special site (impurity atoms, defect clusters) as the nucleation site of the crystal. The nature and efficiency of the nucleation site may depend on:

i) The nature of the incident particle: according to [15], charged particle damage (high transmitted energy, low particle flux) is characterized by a small number of large disordered regions superimposed upon a larger relative number of small clusters of defects, whereas almost all primary damage due to electron irradiation (low transmitted energy, high electron flux) is in the form of single interstitial vacancy pairs.

ii) The purity of the material: the material used in the electron irradiation experiments was $\mathrm{ZnO}$ films prepared in the form of ribbons thin enough for electron microscope observations, by hydrolytic reaction of $\mathrm{ZnS}$ vapor at $1100^{\circ} \mathrm{C}$ [13-14]. The material used in the present investigation was bulk doped $\mathrm{ZnO}$.

iii) The irradiation temperature.

iv) The diffusion processes which are certainly different in thin films and in bulk crystals.

Finally, one cannot exclude the possibility that the $1 \Delta$ loops observed after Ar ion bombardment are not in the equilibrium configuration.

\section{Appendix}

\section{On the Relative Stability of $1 \Delta$ and $3 \Delta$ Faulted Loops}

The total energy of a faulted loop includes both the elastic self energy of the dislocation loop $W_{1}$ and the surface energy of the enclosed stacking fault (S.F.). For a $3 \Delta$ faulted loop of radius $r$ (Burgers vector $c / 2=1 / 2[0001]$ normal to the fault plane) it may be written as:

$$
W_{3 \Delta}=\pi r^{2} \gamma_{3 \Delta}+W_{1}(c / 2)
$$

where $\gamma_{3 \Delta}$ is the S.F. energy of the $3 \Delta$ fault and $W_{1}(c / 2)$, the self energy of the corresponding Frank loop.

Sweeping the $3 \Delta$ faulted loop by a glissile Shockley partial (Burgers vector $p=1 / 3\langle 01 \overline{1} 0\rangle$ ) leads to the formation of $1 \Delta$ faulted loop, whose energy is then:

$$
W_{1 \Delta}=\pi r^{2} \gamma_{1 \Delta}+W_{1}(c / 2)+W_{1}(p)
$$

where $\gamma_{1 \Delta}$ is the S.F. energy of the $1 \Delta$ fault and $W_{1}(p)$ is the self energy of the Shockley loop.

In writing this expression, it is implicitly assumed that there is no elastic interaction between the Frank and Shockley loops, which is certainly a correct approximation since their Burgers vectors are perpendicular.

Then the relative stability of both faulted loops depends on the difference

$$
W_{3 \Delta}-W_{1 \Delta}=\pi r^{2}\left(\gamma_{3 \Delta}-\gamma_{1 \Delta}\right)-W_{1}(p)
$$

In the frame of the isotropic elasticity, the self energy of a glissile loop with Burgers vector $p$ may be written [16]:

$$
W_{1}(p)=2 \pi r \mu[1+1 /(1-\nu)]\left(p^{2} / 8 \pi\right) \ln [(4 r / \rho)-2]
$$

with $\mu$ : shear modulus, $\nu$ : Poisson ratio, $\rho$ : core parameter. 
However, hexagonal crystals are isotropic in the basal plane. This makes it possible to take anisotropy into account in the calculations. As shown by Hirth and Lothe [16], the energy expression of the isotropic theory is correct with a particular choice for $\mu$ and $\mu /(1-\nu) ; \mu=K_{\mathrm{s}}$, $\mu /(1-\nu)=K_{\mathrm{e}}$, where $K_{\mathrm{s}}$ and $K_{\mathrm{e}}$ depend on the elastic constants of the material. So, in the frame of the anisotropic elasticity theory, $W_{1}(p)$ may be written as $(p=a / \sqrt{3}, a$ : lattice parameter):

$$
W_{1}(p)=\left(a^{2} r / 12\right)\left(K_{\mathrm{e}}+K_{\mathrm{s}}\right) \ln (4 r / \rho-2)
$$

Introducing $W(R)=\left(W_{3 \Delta}-W_{1 \Delta}\right) / \pi a^{3}$, one has

$$
W(R)=\Gamma R^{2}-\alpha R \ln \beta R, \text { where } R=r / a .
$$

$\Gamma=\left(\gamma_{3 \Delta}-\gamma_{1 \Delta}\right) / a$ depends on the S.F. energies

$\alpha=\left(K_{\mathrm{e}}+K_{\mathrm{s}}\right) / 12 \pi$ depends on the elastic constants of the material

$\beta=4 a / \rho e^{2}$ ( $e$ : basis of neperian logarithms), depends on the dislocation core radius.

Calculations indicate that:

i) when $\Gamma>\Gamma_{\mathrm{c}}=\alpha \beta / e, W(R)$ is always $>0$, that is the $1 \Delta$ faulted loop is always stable.

ii) when $\Gamma<\Gamma_{\mathrm{c}}$, only loops with $R$ greater than a critical value $R_{\mathrm{c}}$ (for which $W\left(R_{\mathrm{c}}\right)=0$ ) are stable in their $1 \Delta$ form $\left(W\left(R>R_{\mathrm{c}}\right)>0\right)$.

For $\mathrm{ZnO}, a=0.3249 \mathrm{~nm}, K_{\mathrm{s}}=4.3410^{10} \mathrm{~N} / \mathrm{m}^{2}$ and $K_{\mathrm{e}}=7.2610^{10} \mathrm{~N} / \mathrm{m}^{2}$ (these values being calculated from the elastic constants given in [17]) and $\left(\gamma_{3 \Delta}-\gamma_{1 \Delta}\right)_{\mathrm{c}}=\gamma_{\mathrm{c}}=a \Gamma_{\mathrm{c}}=370 \beta\left(\mathrm{mJ} / \mathrm{m}^{2}\right)$.

It is seen that $\gamma_{c}$ strongly depends on the core parameter, the correct value of which is still debated. A lower limit of $\beta$ is certainly $\beta=1$, so that $\gamma_{c}>370 \mathrm{~mJ} / \mathrm{m}^{2}$. For lower values of $\left(\gamma_{3 \Delta}-\gamma_{1 \Delta}\right)$, the stability of the loops depends on their size. As an example, for $\left(\gamma_{3 \Delta}-\gamma_{1 \Delta}\right)=$ $100 \mathrm{~mJ} / \mathrm{m}^{2}$, the critical radius $r_{\mathrm{c}}=a R_{\mathrm{c}}$ above which $1 \Delta$ loops are energetically stable is (with $\beta=1) r_{\mathrm{c}}=12 \mathrm{~nm}$.

\section{References}

[1] Sinclair R., Ponce F.A., Bravman J.C., Yamashita T. and Pirouz P., in: "Microscopy of Semiconducting Materials", A.G. Cullis and D.C. Joy Eds. (The Inst. Phys. Bristol and London, 1981) 147.

[2] Yamashita T., Ponce F.A., Pirouz P. and Sinclair R., Philos. Mag. A 45 (1983) 693.

[3] Echigoya J., Pirouz P. and Edington J.W., Philos. Mag. A 45 (1982) 455.

[4] Suzuki K., Takeuchi J., Shino M., Kanaya K. and Iwanaga H., Techn. Report of ISSP Ser. A (1983) $\mathrm{N}^{\circ} 1304$.

[5] Clarke D.R., J. Appl. Phys. 49 (1978) 2407.

[6] Suyama Y., Tomokiyo Y., Manabe T. and Tanka E., J. Am. Ceram. Soc. 71 (1988) 391.

[7] Jong Choul Kim and Goo E.J., Am. Ceram. Soc. 73 (1990) 877.

[8] Amelinckx S., "Dislocations in particular structures", in Dislocations in Solids, Vol. 2, F.R.N. Nabarro Ed. (North-Holland Publishing Co, 1982) 171.

[9] Peigney A., Couderc J.J., Demai J.J., Carles V. and Rousset A., Electron Microscopy 1994, ICEM-13 (Ed. de Physique, Paris, 1994) 847.

[10] Takeuchi S., Suzuki K. and Maeda K., Philos. Mag. A (1984) 171.

[11] Iwanaga H., Suzuki K. and Takeuchi S., Philos. Mag. 34 (1976) 291.

[12] Iwanaga H., Shibata N., Suzuki K. and Takeuchi S., Philos. Mag. 35 (1977) 1213.

[13] Yoshiie T., Iwanaga H., Shibata N., Suzuki K., Ichihara M. and Takeuchi S., Philos. Mag. A 47 (1983) 315.

[14] Yoshiie T., Iwanaga H., Shibata N., Suzuki K. and Takeuchi S., Philos. Mag. A 41 (1980) 935.

[15] Crawford J.H., The Interaction of Radiation With Solids, R. Strumane, J. Nihoul, R. Gevers and S. Amelinckx Eds. (North Holland, Amsterdam, 1964) 421.

[16] Hirth J.P. and Lothe J., Theory of Dislocations (McGraw-Hill, N.Y., 1968).

[17] Bateman T.B., J. Appl. Phys. 33 (1962) 3309. 